Reading comprehension and vocabulary: Is vocabulary more important for some aspects of

comprehension?

Kate Cain*

Lancaster University

Jane Oakhill

University of Sussex

*corresponding author

Address for correspondence

Department of Psychology

Fylde College

Lancaster University

Lancaster

LA1 4YF

Email: k.cain@lancaster.ac.uk

Phone: +44 (0)1524 593990

Cain, K., \& Oakhill, J. (2014). Reading comprehension and vocabulary: Is vocabulary more important for some aspects of comprehension? L'Année Psychologique, 114, 647-662. 


\begin{abstract}
The influence of vocabulary breadth (number of words known) and vocabulary depth (what is known about those words) on different aspects of text comprehension was examined in 8310 - to 11-year-olds. Vocabulary was not an important predictor of comprehension for details explicitly stated in the text. In contrast, vocabulary was related to inference making and, in particular, measures of vocabulary that assessed what was known about individual words predicted unique variance in global coherence inferences. The pattern of findings supports previous research reporting that measures of breadth and depth of vocabulary knowledge are important predictors of both reading comprehension and comprehension-related skills, and critically identifies that some aspects of vocabulary knowledge may be more important for higher-level comprehension skills than others.
\end{abstract}

Keywords: vocabulary, inference making, text comprehension, lexical quality 
Reading comprehension and vocabulary: Is vocabulary more important for some aspects of comprehension?

Adults' and children's vocabulary knowledge is strongly associated with their reading comprehension ability (Carroll, 1993) and, clearly, adequate comprehension of a text could not take place without an understanding of the individual words. Here, we consider vocabulary within the context of Perfetti's (2007) lexical quality hypothesis, that is we are concerned about the quality of word knowledge, in addition to the number of words known. Broadly speaking, the number of words known is considered a measure of vocabulary breadth. In contrast, what an individual knows about those words and their semantic associates is considered a measure of depth or quality of knowledge. Research with children to date has found that both breadth and depth of vocabulary knowledge are predictive of reading comprehension ability (Oakhill \& Cain, 2012; Ouellette, 2006; Roth, Speece, \& Cooper, 2002; Tannenbaum, Torgesen, \& Wagner, 2006). Our unique perspective is to consider how vocabulary is related to different aspects of reading comprehension, namely memory for the text (literal comprehension) and inference making. In this study, we consider the role of these two aspects of vocabulary knowledge (breadth and depth) in relation to literal comprehension and different types of inference making, to gain a more detailed understanding of the role of vocabulary in children's text comprehension. As a framework, we first consider the roles of integration and inference in text comprehension and review research on individual differences in these processes. This is followed by a review of research on the relation between vocabulary, inference, and reading comprehension. 


\section{The role of inference in text comprehension}

There is a strong consensus that readers and listeners go beyond the literal details of a text and construct a memory-based representation of the state of affairs described in the text, referred to as a mental model (Johnson-Laird, 1983) or a situation model (Kintsch, 1998). When constructing this model, skilled comprehenders strive for cohesion and coherence (van den Broek, Risden, \& Husebye-Hartman, 1995): they integrate the ideas presented in the text and generate inferences using their background knowledge. There are many different taxonomies of inference but no consensus on their classification (Graesser, Singer, \& Trabasso, 1994). For our research questions, the most relevant framework is to distinguish between local cohesion inferences and global coherence inferences (Graesser et al., 1994), also referred to as text-connecting (or bridging) and gap-filling inferences in the previous literature (Baker \& Stein, 1981; Cain \& Oakhill, 1999; Elbro \& Buch-Iverson, 2013; Graesser et al., 1994; Halliday \& Hasan, 1976; Trabasso \& Suh, 1993). We discuss each of these two types in turn, below.

Text integration is essential to construct a mental model. As each new sentence or proposition is processed, skilled comprehenders attempt to integrate its meaning with their current model. Local cohesion inferences enable the reader to go beyond the surface or literal meaning to establish connections between different propositions. This network of connected propositions is called the textbase level of representation (Kintsch \& Rawson, 2005). Individual differences in the ability to integrate propositions, generating inferences where necessary to achieve this, is related to both children's and adults' text comprehension. For example, Oakhill 
(1982) demonstrated that children with good text comprehension are more likely to integrate sentences such as 'The mouse ate some food. The food was bread.' than poor comprehenders. As a result, good comprehenders are more prone to false recognitions of sentences that combine this information, such as 'The mouse ate some bread'.

In general, propositional integration is not signalled by noun phrase repetition as above (some food - the food), but requires a mapping between related words, for example between synonyms or category-exemplar pairings as in 'He fetched a glass of orange juice. The drink was very refreshing.' Successful integration in this example requires an inference that draws on vocabulary (or background) knowledge, in addition to integrative processing. Again, children who are good text comprehenders are more likely to make these local cohesion inferences than children with poorer general text comprehension ability (Cain \& Oakhill, 1999). The essence of these inferences is that they are activated by single words or phrases and enable the integration of the meanings of separate propositions in the text.

For adults, there are clear ability differences in the time course of this integration process. When reading the sentence pair: "Allen's baby became violently ill, so Allen got the baby in the car and rushed off to the emergency room. The hospital had a long waiting line." skilled adult readers show an ERP signal indicative of immediate integration when reading 'hospital' (Perfetti, Yang, \& Schmalhofer, 2008). Less skilled adult readers do not show this signal. Perfetti and colleagues suggest that individual differences in the quality of semantic representations underpin this effect: 
skilled readers have higher quality lexical representations that influence the speed of their word retrieval and integrative processes.

Not all details are stated explicitly in a text; readers typically have to engage in inference making to fill in this missing detail. Global coherence inferences draw on vocabulary and general knowledge to make full sense of details that have been left unstated. An example of this type of inference is establishing the setting of a story when the location is not explicitly stated but can be inferred by identifying the links between semantically related concepts such as 'building sandcastles', 'paddling in the water', and the presence of a 'pier' (which together indicate that the setting is the seaside). These inferences differ from local cohesion inferences in that they are not signalled by a single word or noun phrase and do not involve the integration of two propositions in the text. Readers can construct an integrated mental model without generating these global coherence inferences, but it would be vague and underspecified if the comprehender did not draw on their rich semantic knowledge networks to make full sense of the text. For that reason, these inferences are considered necessary for adequate text comprehension (Graesser et al., 1994) and differ from purely elaborative inferences, which are not required for global coherence. Children with good text comprehension are more likely to generate global coherence inferences than poorer comprehenders (Cain \& Oakhill, 1999; Oakhill, 1984). Poor comprehenders' difficulties with these inferences are related to the inference making process, rather than lack of relevant knowledge: when asked directly, they demonstrate that they do have the requisite background knowledge (Cain \& Oakhill, 1999). 
Inferential processing that is dependent on general knowledge has been studied in adults

to determine if they use context to select the appropriate sense of an ambiguous word, as in: 'The townspeople were amazed to find that all the buildings had collapsed except the mint'. Lessskilled adult readers are less likely to draw on their general knowledge spontaneously to make such inferences (Long, Oppy, \& Seely, 1994). As in the case of children, this effect may not be through lack of knowledge: when prompted, less-skilled adult readers do make such inferences (Hannon \& Daneman, 1998). These findings link back to Perfetti's ideas about lexical quality and accessibility of rich semantic representations of words, although Perfetti has not to date investigated how lexical quality is specifically related to global coherence inferences.

\section{The relation between vocabulary and inference making}

Our analysis of the skills involved in integrative and inferential processing confirms the importance of one type of knowledge - vocabulary - for text comprehension and inference in particular. As Kintsch and Rawson (2005) stated: 'Comprehension requires inferences, and inferences require knowledge' (p. 221). Our analysis also indicates that good vocabulary knowledge may be more important for global coherence inferences than for local cohesion inferences, because having rich, detailed and precise semantic representations of words makes it more likely that thematically-related inferences will be made to establish coherence. Such inferences are not triggered by "cohesive ties", such as anaphors, and so are likely to be more dependent on such activation processes.

Previous research investigating the relation between vocabulary and reading 
comprehension has measured vocabulary breadth and depth in different ways: in particular, word definitions has been included as a measure of breadth (Tannenbaum et al., 2006) as well as depth (Ouellette, 2006). We believe the ability to define a word is an aspect of vocabulary depth because it concerns 'how well the meanings are known' (Ouellette, 2006) and 'the richness of knowledge that the individual possess about the words that are known' (Tannenbaum et al., 2006, p. 383). As noted by others, there are different degrees of depth of knowledge including being able to provide a detailed definition of a word and understanding the relations between that word and others (Tannenbaum et al., 2006). This contrasts with vocabulary breadth, which refers to the size of the lexicon (Ouellette, 2006; Tannenbaum et al., 2006), and is typically assessed with single-word recognition tasks as an estimate of the size of the lexicon (Ouellette, 2006).

Previous research has demonstrated that measures of vocabulary that tap an individual's ability to define words or understand the interrelations between words are more strongly related to general measures of reading comprehension than other measures of vocabulary knowledge, although both are predictive of comprehension level (Ouellette, 2006; Tannenbaum et al., 2006). However, the extent to which these two aspects of vocabulary knowledge are related to reading comprehension may depend on the nature of the reading comprehension assessment, which can differ on many dimensions including the extent to which literal and inferential comprehension are assessed (Cain \& Oakhill, 2006). Given the central importance of literal memory, local cohesion inferences, and global coherence inferences to the construction of an adequate mental model of a text, it is important to determine how vocabulary knowledge is related to each. 
Current study. We analyse data collected as part of a longitudinal investigation of the predictors of reading comprehension (Oakhill \& Cain, 2012) to determine whether vocabulary breadth and depth each contribute to different types of inference making and literal comprehension. If vocabulary knowledge makes a specific contribution to the inference making process it should predict inference making over and above word reading ability (as found for reading comprehension by Ouellette, 2006) and also over and above literal memory for the text. Further, for the reasons outlined above, we predicted that vocabulary would be more important for global coherence inferences than local cohesion inferences, and that depth of vocabulary knowledge would predict variance over and above breadth of vocabulary knowledge for this type of inference. When the sample was aged 10 to 11 years, they completed oral assessments of vocabulary that tapped breadth and depth of vocabulary knowledge. The children also completed an experimental assessment of inference making, in which they read short stories and then answered questions tapping: recall of literal details in the text; the ability to integrate propositions within the text (local cohesion inferences); and the ability to work out settings and events in the story, which were not explicitly stated (global coherence inferences).

\section{Method}

\section{Participants}

The sample comprised 83 children aged 10 to 11 years ( $M=10$ years, 7 months, range: 122-133 months; 47 girls, 36 boys). The children came from schools in a range of middle to lower class catchment areas in the south of England. The sample was originally selected 4 years earlier 
for a longitudinal study (Oakhill \& Cain, 2012) and came from nineteen classrooms across five schools, ensuring a wide range of ability and home backgrounds. At the outset of the study, when the children were aged 7 to 8 years, they were selected to have word reading that was not more than two years or more below chronological age to ensure that word reading was sufficient for the full battery of assessments, and also to have word reading that was not more than 2 years above chronological age to ensure that reading ability would be within the scale of the test used to measure reading ability four years later. All children spoke English as their first language and none had reported behavioural, emotional, or learning difficulties. The gender balance reflected the proportion of girls and boys who met the exclusion criteria.

\section{Ability assessments and procedure}

Reading ability. All children completed the Neale Analysis of Reading Ability: revised (NARA-R: Neale, 1989), in which children read short passages and answer open-ended questions after each one. We report the word reading accuracy scores, which are based on the number of word reading errors made, and reading comprehension scores, which are based on the number of questions about the stories that children answer correctly. Children completed Form 1, for which the reliability reported in the manual (test-retest) for this age group is .86 for word reading accuracy and .82 for reading comprehension.

Vocabulary. Our measure of vocabulary breadth was the British Picture Vocabulary Scales (BPVS: Dunn, Dunn, Whetton \& Pintillie, 1992), in which children have to select one out of four pictures that represents the meaning of a word spoken by the experimenter. Thus, this estimates 
the number of entries in the lexicon rather than in-depth knowledge of those words. Testing stops when a prescribed number of errors has been made. The reliability reported in the manual (Median Cronbach's $\alpha$ ) is .93. The American version of this test has been used to assess vocabulary breadth in previous research (Ouellette, 2006; Tannenbaum et al., 2006).

There is currently no consensus on how to measure vocabulary depth (compare (Ouellette, 2006; Tannenbaum et al., 2006), so we included two measures of this construct to reflect different levels of knowledge of word meanings in order to obtain a more reliable estimate. These were the Vocabulary and Similarities subtests from the Wechsler Intelligence Test for Children - III (WISC-III: UK edition, Wechsler 1992). The Vocabulary subtest requires participants to define words that increase in difficulty, for example 'alphabet', 'island', 'precise', and the Similarities subtest requires participants to identify how two things are similar, for example 'wheel' and 'ball' (easy item) and 'first - last' (more difficult item) and, thus, taps the richness of knowledge about the words that are known. The reliabilities reported in the manual (split-half reliabilities) for this age group are .88 and .82 , respectively.

All tests were scored in accordance with the manual. Raw scores were used in the analysis for all measures with the exception of vocabulary depth. For these measures, we could not use raw scores because there were different numbers of items in the two subtests. Instead, we calculated the percentage correct over the maximum score possible for each subtest and calculated the average of the two percentages. 
Inference and integration task. We developed a bespoke measure of comprehension skill using the materials of Cain and Oakhill (1999) as a template. The assessment comprised three short stories, each followed by questions that tapped either memory for literal information in the text, the ability to generate local cohesion inferences by integrating two premises in the text, or the ability to general global coherence inferences by integrating textual premises with general knoweldge. The information required to answer a literal question was stated explicitly in the text. For example "She left a puddle of water in the kitchen by the fridge where she had been standing" provides the answer to the question "Where was the puddle of water?". The information required to answer a local cohesion inference question was provided in two adjacent sentences. For example: "Mum looked for the cleaning equipment. She found the bucket in the cupboard under the stairs." was the information that provided the answer to the question "Where did mum look for the cleaning equipment?" The information required to answer a question tapping global coherence required the reader to integrate information in the text with general knowledge. For example the text "Jake watched a small creature close by. He threw over some crumbs, but that was not what the little creature wanted. It hopped into the water and swam away." provides the information to answer the question "What sort of animal did Jake try to feed?".

Children read three short passages aloud and were then asked open-ended questions after each one. There were two questions for each question type and one point was awarded for each question (maximum score for literal = 6; maximum score for local cohesion inferences = 6; maximum score for text with global coherence inferences $=6$ ). Responses were scored using a 
rubric of correct and incorrect answers. There were too few items to obtain a reliable measure of internal consistency for each question type, but the overall internal consistency for this measure was $\alpha=.61$.

\section{Results}

An examination of skew and kurtosis revealed that all variables were within acceptable limits with the exception of performance on the literal questions on the inference task, which were negatively skewed and approaching ceiling performance.

The descriptive statistics and zero-order correlations are reported in Table 1. The correlations revealed that none of the vocabulary measures was associated with recall of literal details, they were weakly associated with the local cohesion inferences, and were most strongly associated with the global coherence inferences. These zero-order correlations were of a similar magnitude. Of note, scores on the local cohesion inference questions were slightly higher (57\% correct) than scores on the global coherence inferences (48\%), but performance on both was variable and children scored from 1 through to maximum marks (6) with only one child obtaining 100\%. In addition, and in line with previous research, both vocabulary and inference making were strongly correlated with reading comprehension, the relationship was weaker between local cohesion inferences and reading comprehension than between global coherence inferences and reading comprehension. 


\section{Are literal memory, local cohesion inferences, and global coherence inferences equally reliant}

\section{on vocabulary knowledge?}

To test our predictions, we performed two pairs of multiple regressions to predict performance on the local cohesion inferences and global coherence inferences. We did not examine prediction of performance on the literal questions because performance on these was near ceiling and this measure did not correlate with vocabulary. Our measure of inference making required children to read the texts themselves, rather than listen to them. Therefore, in the first step for all analyses, word reading accuracy as well as age was entered to control for differences in word reading ability. In one analysis for each criterion, vocabulary breadth was entered in the second step and vocabulary depth was entered in the third step (Model 1 in Table 2). In the complementary analysis of the pair, the order of vocabulary breadth and depth was reversed

(Model 2 in Table 2). This procedure enabled us to identify whether each dimension of vocabulary predicted unique variance in performance on the different question types. We further determined the relative contributions of each vocabulary measure by examining the standardised Beta coefficients for the final model.

The results of the analyses for the two inference types are reported in the top half of Table 2. Vocabulary depth and breadth each predicted variance in the local cohesion inferences when entered in the second step, but neither predicted additional variance when entered in the third step, that is after the other measure of vocabulary (compare Models 1 and 2). In contrast, vocabulary depth was a significant predictor of variance in performance on the global coherence 
inference questions when entered after vocabulary breadth. Comparison of the standardised beta coefficients confirmed the above pattern of prediction: neither vocabulary breadth nor depth predicted significant unique variance in local cohesion inferences; in contrast, vocabulary depth was a significant unique predictor of global coherence inferences. In addition, together the vocabulary measures explained a greater proportion of variance in global coherence inferences $\left(R^{2}\right.$ $=.25)$ than in local cohesion inferences $\left(R^{2}=.07\right)$. Although these two aspects of vocabulary knowledge are related (see Table 1), these analyses provide some indication that they tap partially separable aspects of vocabulary knowledge. In general, vocabulary was important for both inference types and predicted significant variance after controlling for age and word reading accuracy. Despite the shared variance between our two measures of vocabulary, vocabulary depth was a significant unique predictor of global coherence inferences.

The zero-order correlations revealed that performance on the literal questions was significantly related to that on the local cohesion inferences $(r=.38)$ with a weaker association $(r$ $=.20$ ) with the global coherence inferences. Therefore, we conducted a subsidiary set of analyses to examine the prediction of the two types of inference skill by vocabulary when also controlling for performance on the literal questions (Model 3 in Table 2). In each analysis, performance on the literal questions was entered in the second step (after age and word reading accuracy) and the two vocabulary measures were entered together in the third and final step. As seen in Table 2 we found that literal memory predicted performance on local cohesion inferences and vocabulary did not make a significant contribution when entered after these scores, although the proportion of 
variance explained by vocabulary in Model 3 was reduced only slightly relative to Models 1 and 2

(4.9\% to $3.8 \%)$. In contrast, literal performance did not predict a statistically significant amount of variance in global coherence inferences, whilst vocabulary depth did predict a statistically significant proportion of unique variance when considered alongside these scores.

Table 2

Prediction of performance on inference questions

\begin{tabular}{|c|c|c|c|c|c|}
\hline \multirow[t]{2}{*}{ Step } & \multirow[t]{2}{*}{ Measures } & \multicolumn{2}{|c|}{$\begin{array}{l}\text { Local cohesion } \\
\text { inferences }\end{array}$} & \multicolumn{2}{|c|}{$\begin{array}{l}\text { Global coherence } \\
\text { inferences }\end{array}$} \\
\hline & & $\Delta R^{2}$ & Final Beta & $\Delta R^{2}$ & Final Beta \\
\hline \multicolumn{6}{|c|}{ Model 1} \\
\hline \multirow[t]{2}{*}{ 1. Controls } & age & .010 & .042 & .047 & -.002 \\
\hline & accuracy & & -.160 & & .016 \\
\hline 2. Vocabulary & breadth & $.062^{*}$ & .201 & $.168 * * *$ & .227 \\
\hline 3. Vocabulary & depth & .010 & .122 & $.085^{* *}$ & $.371 * *$ \\
\hline \multicolumn{6}{|c|}{ Model 2} \\
\hline 2. Vocabulary & depth & $.049^{*}$ & .122 & $.224 * * *$ & $.371^{* *}$ \\
\hline 3. Vocabulary & breadth & .023 & .201 & .029 & .227 \\
\hline \multicolumn{6}{|c|}{ Model 3} \\
\hline 2. Literal & literal & $.139 * * *$ & $.338 * *$ & $.051^{*}$ & .134 \\
\hline \multirow[t]{2}{*}{ 3. Vocabulary } & breadth & .038 & .140 & $.219 * * *$ & .203 \\
\hline & depth & & .100 & & $.362 * * *$ \\
\hline \multicolumn{6}{|c|}{ Model 4} \\
\hline 1. Control & age & .008 & .061 & .003 & -.004 \\
\hline \multirow[t]{2}{*}{ 2. Vocabulary } & breadth & .052 & .012 & $.296 * * *$ & .233 \\
\hline & depth & & .014 & & $.372 * * *$ \\
\hline
\end{tabular}

Note. $\Delta R^{2}$ is the value for that step; Final Beta is reported as the standardised coefficient for each individual measure. Accuracy $=$ Word reading accuracy; Literal $=$ Literal questions. ${ }^{*} p<.05 ;{ }^{* *} p$ $<.01 ; * * * p<.001$ 
As shown by the zero-order correlations (Table 1) and the multiple regression analyses

(Table 2, Models 1-3), vocabulary breadth and depth were more strongly correlated with global coherence inferences than with local cohesion inferences. However, vocabulary breadth was itself more strongly correlated with word reading accuracy than was vocabulary depth, which is the same pattern as that found by Ouellette (2006). Therefore, in a final set of analyses (Table 2, Model 4), we did not include word reading accuracy as a control in order to determine the full predictive contribution of vocabulary to inference making. Similar to the other models, vocabulary breadth did not predict significant variance in either local cohesion nor global coherence inferences.

\section{Discussion}

Our analyses elucidate the relations between vocabulary knowledge and text comprehension in young readers in three important ways. First, we have demonstrated that vocabulary may be more important for some aspects of comprehension than others; specifically vocabulary was more important for inference making than for literal memory for the text. Second, we have shown that vocabulary in general predicts a greater proportion of variance in global coherence inferences than in local cohesion inferences. Third, we have shown that depth, rather than breadth, of vocabulary knowledge is the more critical factor in explaining performance on global coherence inferences, even after memory for the text and word reading are taken into account. The pattern of difference reported here provides new information about the role of 
lexical quality in children's and adults' comprehension (Perfetti \& Stafura, 2014; Richter, Isberner, Naumann, \& Neeb, 2013).

As predicted, vocabulary knowledge was more strongly related to inference making than to literal comprehension of the text. This contrast was not unexpected because the individual words in the text were selected to be age appropriate and, thus, variation in vocabulary knowledge would not be expected to play a strong role in memory for explicit details in the text. The moderate to strong relationships between our inference measures and the general measure of reading comprehension (NARA) validates our inference task as a core component of good text comprehension. Our focus in this set of analyses was to determine the role played by vocabulary in a core reading comprehension skill - inference. Thus, the contrast between the strength of the relation between vocabulary and the two different inference types requires more detailed consideration.

Previous research has established the importance of vocabulary knowledge in word to text integration in adults (Perfetti et al., 2008), which is closely aligned with the importance of breadth of knowledge for the local cohesion inferences in the present study, since both processes are activated by single words. We have demonstrated that vocabulary is important in this process in children, predicting significant variance in local cohesion inferences when entered after age and word reading ability. However, vocabulary was not a significant predictor of local cohesion inferences when entered after literal memory for the text. One reason for the strength of the prediction by literal memory is that these inferences require the reader to establish links between 
different propositions in the text and an accurate representation of the text's meaning is particularly important when doing so.

On first glance, it is surprising that vocabulary was not a significant predictor of performance on local cohesion inferences when entered after literal memory because this inference type requires the comprehender to identify (or know) a category exemplar or synonym. It should be noted that our measures were not timed and this might explain why vocabulary was not strongly predictive of this type of inference. Recent work has shown that accuracy of word knowledge and speed of retrieval make independent contributions to concurrent text comprehension (Oakhill, Cain, McCarthy, \& Nightingale, 2012; Richter et al., 2013), suggesting that these measures tap different aspects of lexical quality. Thus we predict that a speeded measure may be more sensitive in picking up the variance associated with the role of vocabulary in integration. A clear message for future research is that measures of both accuracy and speed to access vocabulary knowledge are needed to understand the role of vocabulary on different types of inference making.

In the case of global coherence inferences, vocabulary, and in particular depth of word knowledge, was found to be a strong predictor of performance. The ability to make global coherence inferences taps interrelations between different words that are associated by setting, theme, or character identity. Thus these inferences place a greater reliance on the richness of an individual's semantic network, which is tapped by measures of vocabulary depth. It seems, therefore, that different types of inference may be more heavily dependent on different aspects 
of vocabulary knowledge: in particular, knowledge about the interrelations between words enhances the ability to generate global coherence inferences. We used a definitions task as one of our depth measures as did Ouellette (2006) and, in accord with Ouellette (2006), we also found that our breadth measure was more strongly correlated with word decoding than vocabulary depth. Thus, our pattern of differential prediction seems robust.

The relation between general verbal ability and comprehension is well noted (Carroll, 1993). However, verbal ability is a broad construct and knowledge of this relation does not tell us which aspects of verbal ability are related to reading comprehension processes such as inference nor why the relation might exist. One implication of our findings is that we have demonstrated that different aspects of vocabulary knowledge, rather than verbal ability more generally, are related to a core aspect of comprehension, namely inference. Thus, our work extends that of Ouellette (2006) and Tannenbaum et al. (2006) by demonstrating a specific relation between vocabulary and inference, but not literal comprehension, which fits well with work on lexical quality and inference in adults (Perfetti et al., 2008).

Clearly, one limitation of this study is that we used only a single measure of vocabulary breadth and two measures of vocabulary depth and some might argue that measurement error may play a role in the differential prediction of these two aspects of vocabulary. We note that in our study, as in others, different measures of vocabulary share variance and are not completely distinct (Ouellette, 2006; Proctor, Silverman, Harring, \& Montecillo, 2012; Tannenbaum et al., 2006). What these and other findings suggest is that it is more theoretically informative to view 
vocabulary as a multidimensional construct in order to under better its relation with higher-level cognition. Future research needs to clarify empirically how best to distinguish and assess different dimensions of vocabulary, to aid future investigations into their precise relation with different aspects of reading. Such studies should include multiple measures of both breadth and depth of vocabulary, to inform latent constructs of these dimensions and, in addition, a broader conceptualisation of vocabulary knowledge might also be achieved by including knowledge of morphology and syntax (Proctor et al., 2012).

Another limitation is our focus on a single age group. Thus our data do not address potential developmental differences in the contribution of vocabulary knowledge, or types of vocabulary knowledge, to inference making. Future work might consider this perspective and also the reciprocal relations that may exist between inference and vocabulary (Cain, Oakhill, \& Lemmon, 2004) and the role that metalinguistic awareness might play in word-learning ability (Nagy, 2007). We should also consider how the ease of our literal questions restricted our ability to examine how vocabulary predicted performance on this aspect of comprehension. Literal comprehension is clearly essential to the construction of a mental model, so a deeper understanding of the factors that influence memory for these stated details in the text would be a useful focus of future work.

This is the first study to explore the relation between different dimensions of vocabulary knowledge on different aspects of text comprehension. This is an opportunistic and small-scale investigation of the relation between different aspects of vocabulary and text comprehension 
factors, albeit one with important findings. Our data are correlational and do not speak to the online process of recruiting vocabulary knowledge and meaning activation during comprehension. However, it is likely that readers with richer vocabulary knowledge would be more likely to use this information as they read to add to details provided by the text. These findings also require validation across a larger age range to establish the generality of these effects. Despite these limitations, these findings point to an important conclusion: not all measures of vocabulary or inference making are the same. Future online work using both accuracy and speeded measures of different aspects of vocabulary knowledge is needed to identify when and how during text processing, vocabulary is critical. 


\section{References}

Baker, L., \& Stein, N. (1981). The development of prose comprehension skills. In C. M. Santa \& B. L. Hayes (Eds.), Children's prose comprehension: Research and practice (pp. 7-43). Newark, DE: IRA.

Cain, K., \& Oakhill, J. (2006). Assessment matters: Issues in the measurement of reading comprehension. British Journal of Educational Psychology, 76, 697-708. doi:

\section{$10.1348 / 000709905 \times 69807$}

Cain, K., Oakhill, J., \& Lemmon, K. (2004). Individual differences in the inference of word meanings from context: the influence of reading comprehension, vocabulary knowledge, and memory capacity. Journal of Educational Psychology, 96, 671-681. doi:

$10.1037 / 0022-0663.96 .4 .671$

Cain, K., \& Oakhill, J. V. (1999). Inference making and its relation to comprehension failure. Reading and Writing. An Interdisciplinary Journal, 11, 489-503. doi: 10.1023/A: 1008084120205

Carroll, J. B. (1993). Human cognitive abilities: A survey of factor-analytic studies. New York: Cambridge University Press.

Elbro, C., \& Buch-Iverson, I. (2013). Activation of background knowledge for inference making: effects on reading comprehension. Scientific Studies of Reading, 17, 435-452. doi: $10.1080 / 10888438.2013 .774005$ 
Graesser, A. C., Singer, M., \& Trabasso, T. (1994). Constructing inferences during narrative text comprehension. Psychological Review, 101, 371-395. doi: 10.1037/0033295X.101.3.371

Halliday, M. A. K., \& Hasan, R. (1976). Cohesion in English. London: Longman.

Hannon, B., \& Daneman, M. (1998). Facilitating knowledge-based inferences in less-skilled readers. Contemporary Educational Psychology, 23, 149-172. doi: 10.1006/ceps. 1997.0968

Johnson-Laird, P. N. (1983). Mental models: towards a cognitive science of language, inference, and consciousness. Cambridge: Cambridge University Press.

Kintsch, W. (1998). Comprehension: A Paradigm for Cognition. New York, NY: Cambridge University Press.

Kintsch, W., \& Rawson, K. A. (2005). Comprehension. In M. J. Snowling \& C. Hulme (Eds.), The science of reading: a handbook (pp. 209-226). London: Blackwell Publishing.

Long, D. L., Oppy, B. J., \& Seely, M. R. (1994). Individual differences in the time course of inferential processing. Journal of Experimental Psychology: Learning, Memory and Cognition, 20, 1456-1470. doi: 10.1037/0278-7393.20.6.1456

Nagy, W. E. (2007). Metalinguistic awareness and the vocabulary-comprehension connection. In R. K. Wagner, A. E. Muse \& K. R. Tannenbaum (Eds.), Vocabulary acquisition: Implications for reading comprehension (pp. 52-77). New York: Guilford Press. 
Oakhill, J. (1984). Inferential and memory skills in children's comprehension of stories. British Journal of Educational Psychology, 54, 31-39. doi: 10.1111/j.2044-

8279.1984.tb00842.x

Oakhill, J., \& Cain, K. (2012). The precursors of reading comprehension and word reading in young readers: Evidence from a four-year longitudinal study. Scientific Studies of Reading, 16(2), 91-121. doi: 10.1080/10888438.2010.529219

Oakhill, J., Cain, K., McCarthy, D., \& Nightingale, Z. (2012). Making the link between vocabulary knowledge and comprehension skill. In A. Britt, S. Goldman \& J.-F. Rouet (Eds.), From words to reading for understanding (pp. 101-114). New York: Routledge.

Ouellette, G. P. (2006). What's meaning got to do with it: The role of vocabulary in word reading and reading comprehension. Journal of Educational Psychology, 98, 554-566. doi: $10.1037 / 0022-0663.98 .3 .554$

Perfetti, C. A., \& Stafura, J. (2014). Reading comprehension: Including word knowledge in a theoretical framework. Scientific Studies of Reading, 18, 22-37. doi:

$10.1080 / 10888438.2013 .827687$

Perfetti, C. A., Yang, C.-L., \& Schmalhofer, F. (2008). Comprehension skill and word-to-text integration processes. Applied Cognitive Psychology, 22, 303-318. doi: 10.1002/acp.1419

Proctor, C. P., Silverman, R. D., Harring, J. R., \& Montecillo, C. (2012). The role of vocabulary depth in predicting reading comprehension among English monolingual and Spanish- 
English bilingual children in elementary school. Reading \& Writing, 25, 1635-1664. doi:

$10.1007 / \mathrm{s} 11145-011-9336-5$

Richter, T., Isberner, M.-B., Naumann, J., \& Neeb, Y. (2013). Lexical quality and reading comprehension in primary school children. Scientific Studies of Reading, 17, 415-434.

doi: $10.1080 / 10888438.2013 .764879$

Roth, F. P., Speece, D. L., \& Cooper, D. H. (2002). A longitudinal analysis of the connection between oral language and early reading. Journal of Educational Research, 95, 259-272. doi: $10.1080 / 00220670209596600$

Tannenbaum, K. R., Torgesen, J. K., \& Wagner, R. K. (2006). Relationships between word knowledge and reading comprehension in third-grade children. Scientific Studies of Reading, 10, 381-398. doi: 10.1207/s1532799xssr1004_3

Trabasso, T., \& Suh, S. (1993). Understanding text: achieving explanatory coherence through on-line inferences and mental operations in working memory. Discourse Processes, 16, 3-34. doi: $10.1080 / 01638539309544827$

van den Broek, P. W., Risden, K., \& Husebye-Hartman, E. (1995). The role of readers' standards for coherence in the generation of inferences during reading. In R. F. Lorch \& E. J. O'Brien (Eds.), Sources of coherence in reading (pp. 353-373). Mahwah, NJ: Lawrence Erlbaum Associates, Inc. 
Table 1

Correlations between variables and means (standard deviations) for all measures

\begin{tabular}{|c|c|c|c|c|c|c|c|}
\hline & 1 & 2 & 3 & 4 & 5 & 6 & Mean (SD) \\
\hline 1. Word reading accuracy & & & & & & & $77.01(11.08)$ \\
\hline 2. Reading comprehension & .200 & & & & & & $21.33(6.44)$ \\
\hline 3. Vocabulary breadth & $.415^{* * *}$ & $.597 * * *$ & & & & & $103.29(12.98)$ \\
\hline 4. Vocabulary depth & $.270 *$ & $.569 * * *$ & $.643^{* * *}$ & & & & $77.00(11.16)$ \\
\hline 5. Literal questions & -.142 & $.348 * *$ & .170 & .126 & & & $5.40(.76)$ \\
\hline 6. Local cohesion inferences & -.052 & $.378 * *$ & $.230 *$ & .207 & $.384^{* * *}$ & & $3.40(1.10)$ \\
\hline $\begin{array}{l}\text { 7. Global coherence } \\
\text { inferences }\end{array}$ & .205 & $.607^{* * *}$ & $.482 * * *$ & $.516 * * *$ & .201 & $.380 * * *$ & 2.87 (1.37) \\
\hline
\end{tabular}

Note. All raw scores with the exception of vocabulary depth (percentage of raw score). ${ }^{*} p<.05 ;{ }^{* *} p<.01 ;{ }^{* * *} p<.001$ 